EPJ Web of Conferences 73, 08001 (2014)

DOI: $10.1051 /$ epjconf/20147308001

(C) Owned by the authors, published by EDP Sciences, 2014

\title{
Hadron physics with GeV photons at SPring-8/LEPS II
}

\author{
M. Niiyama ${ }^{a}$ for the LEPS II collaboration \\ Dept. of Physics, Kyoto University. Oiwake-cho, Sakyo, Kyoto, Japan
}

\begin{abstract}
A new GeV-photon beamline at SPring-8, LEPS II, has been constructed, and we have successfully observed the first beam at LEPS II in early 2013. The commissioning run using a photon detector has also started, and the detector development for a large solenoid magnetic spectrometer is underway. Physics motivations and the current status of LEPS II are described in this article.
\end{abstract}

\section{Introduction}

In this section, physics motivations for a new GeV-photon beamline at SPring-8 (LEPS II) are described with some important results from the current LEPS experiment. The LEPS collaboration has started physics data taking since 2000. The photon beam of LEPS is produced by the backward Compton scattering (BCS) process of laser photons on 8-GeV electrons of SPring-8. One of the advantages of the BCS photon beam is its high polarization. Laser photons are linearly polarized spontaneously, and the BCS process keeps polarization depending on the scattering angle. We can easily control the direction of polarization of laser photons or change to circular polarization using $\lambda / 2$ or $\lambda / 4$ devices, respectively. Thus, the LEPS provides a linearly or circularly polarized photon beam with a polarization degree of more than $90 \%$ [1]. The polarization observables are powerful tools to investigate the mechanisms of hadron photoproduction, as discussed in the following section.

The LEPS detector is optimized to detect particles produced at forward angles, where mesons produced by the $t$-channel reaction can be measured. The spin density matrix of meson production can be extracted from the angular distribution of meson decay with respect to the linear polarization direction of the photon beam. This observable is sensitive to the spin-parity of a particle which is exchanged in the $t$-channel (parity filter) [2]. The LEPS collaboration has measured the spin density matrix of the $K^{0}(890) \Sigma^{+}$photoproduction at forward angles, and the result indicates a substantial contribution of natural parity, $\kappa$-meson, exchange [3].

One of the physics motivations of LEPS II is to study the $\Lambda(1405)$. The $\Lambda(1405)$ is a mysterious particle. It is assigned as a $\mathrm{P}$-wave excitation of the $\Lambda(1116)$, but its mass is too light compared to other P-wave baryons such as $\Lambda(1520)$ and $N(1535)$ and is difficult to be described by the quark model. The $\Lambda(1405)$ is a candidate of the hadron molecule [4-6], but its internal structure has not been understood well. Recently, the photoproduction of $K^{+} \Lambda(1405)$ has been measured firstly by LEPS [7] followed by

\footnotetext{
ae-mail: niiyama@scphys.kyoto-u.jp
}

This is an Open Access article distributed under the terms of the Creative Commons Attribution License 4.0, which permits unrestricted use, distribution, and reproduction in any medium, provided the original work is properly cited. 
CLAS with higher statistics [8]. At the LEPS II a high intensity and highly polarized photon beam with the energy up to $2.9 \mathrm{GeV}$ is available, and the spin density matrix of $K^{+}(890) \Lambda(1405)$ production can be investigated. In this reaction, we can control the spin-parity of the exchanged particle in the $t$-channel by selecting decay angle of $K^{+}(890)$ with respect to the linear polarization of the incident photon similarly to $K^{0}(890) \Sigma^{+}$production. By selecting unnatural-parity $\left(K^{-}\right)$exchange, we can study the lineshape of the $\Lambda(1405)$, which is expected to shift to heavier mass and narrower width compared to those measured by $K^{+} \Lambda(1405)$ photoproduction [9]. For this measurement, all decay products from $K^{+}(890) \rightarrow K_{s}^{0} \pi^{+}$ and $\Lambda(1405) \rightarrow \pi^{0} \Sigma^{0}$ must be measured, and thus, a large solid angle charged and photon detector is necessary.

Another important result from LEPS is a search for a penta-quark candidate, $\Theta^{+}$, which consists of $u u d d \bar{s}$ quarks. Evidence of the $\Theta^{+}$has been reported by LEPS for the first time in 2003 [10], followed by second positive results in 2009 [11]. In second results, the $\gamma d \rightarrow K^{+} K^{-} p n$ reaction has been analyzed by identifying a quasi-free nucleon reaction inclusively from a missing mass $M M_{N}\left(\gamma, K^{+} K^{-}\right)$and by assuming a spectator nucleon has the minimum momentum. This assumption is a good approximation to minimize a Fermi motion effect. The CLAS collaboration also searched for $\Theta^{+}$in $\gamma d \rightarrow K^{+} K^{-} p n$ reaction by measuring $K^{+} K^{-}$and proton in the final state, however no peak structure was observed [12]. At LEPS, we took 2.6 times larger data in 2006-2007 using the almost same experimental setup of our second report. We have improved our analysis to reject a proton exclusively in the final state (exclusive analysis). Since we used a deuteron target, an incident photon can interact with a neutron or a proton. $\Theta^{+}$can be produced in the $\gamma n \rightarrow K^{-} \Theta^{+} \rightarrow K^{-} K^{+} n$ reaction where a proton is a spectator and remains in the target. On the other hand, in the $\gamma p \rightarrow K^{-} K^{+} p$ reaction where $\Theta^{+}$ production is prohibited, a proton can escape from the target and hit the detector. Thus, we can improve a signal-to-noise ratio by rejecting events with a proton in the final state. In the exclusive analysis, a peak structure was appeared in the invariant mass spectrum of $K^{+} n$ after rejecting proton contributions. The details of the exclusive analysis can be found elsewhere [13]. In addition, we have started further data taking with a setup which has higher proton rejection efficiency. In order to establish $\Theta^{+}$, we must understand the positive result from LEPS and negative one from CLAS consistently. The LEPS detector measures kaons only in the forward region, while the CLAS detector covers large angle region. If the production rate of $\Theta^{+}$strongly depends on the angle, both two results can be understood consistently. For this purpose, a large solid angle detector which covers very forward to backward is necessary.

\section{Status of the LEPS II project}

A new GeV-photon beamline (LEPS II) has been constructed at SPring-8 utilizing a long straight section of the electron storage ring, which has an excellent beam emittance. Thanks to the quality of the electron beam, the angular divergence of BCS photon beam is enough narrow to be guided to $150 \mathrm{~m}$ downstream and to be incident on the target. A new experimental hall has been constructed out of the SPring- 8 storage ring building, where large solid angle detectors can be installed. The intensity of the photon beam is also upgraded to be 10 times higher than that of LEPS by injecting four lasers simultaneously and by focusing the laser to match to the shape of the $8-\mathrm{GeV}$ electron beam. We have successfully observed the first beam in January 2013. Figure 1a shows energy spectra of the photon beam, which were measured using an electromagnetic calorimeter. The black and blue histograms show spectra with and without the laser injection. A clear Compton spectrum (black histogram) which shows the Compton edge at $2.4 \mathrm{GeV}$ can be seen with the laser injection, while a low intensity Bremsstrahlung spectrum (blue histogram) is visible without the laser, which is produced by the interaction of the electron beam with the gas in the storage ring. The spatial distribution of the BCS photon beam in the experimental hall is shown in Fig. 1b. The beam size was confirmed to be quite small at the target which is located $\sim 150 \mathrm{~m}$ downstream from the BCS interaction point. 

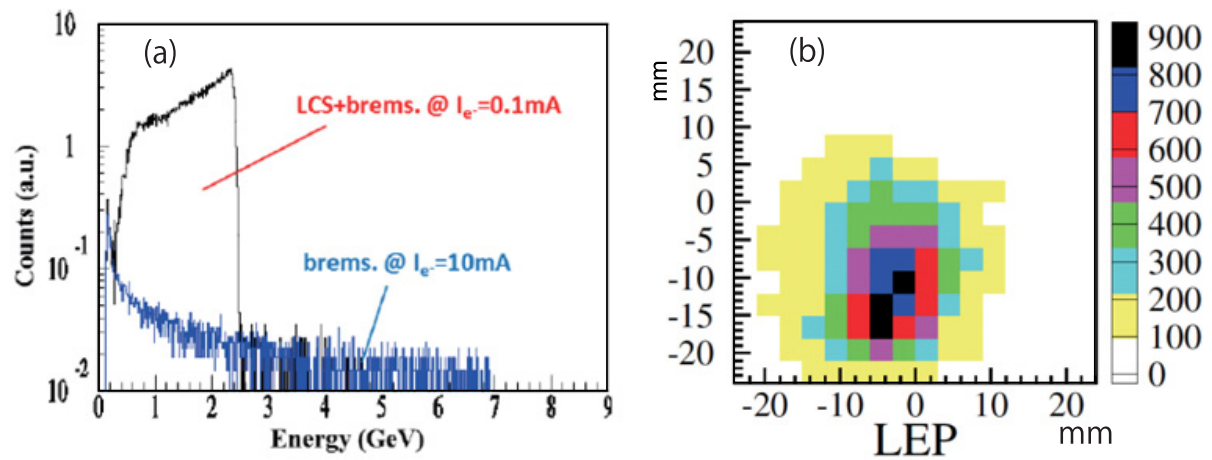

Figure 1. (a) Energy spectra of photon beam at LEPS II. (b) A spatial distribution of BCS photon beam at the target.

\section{(a) BGO EGG}

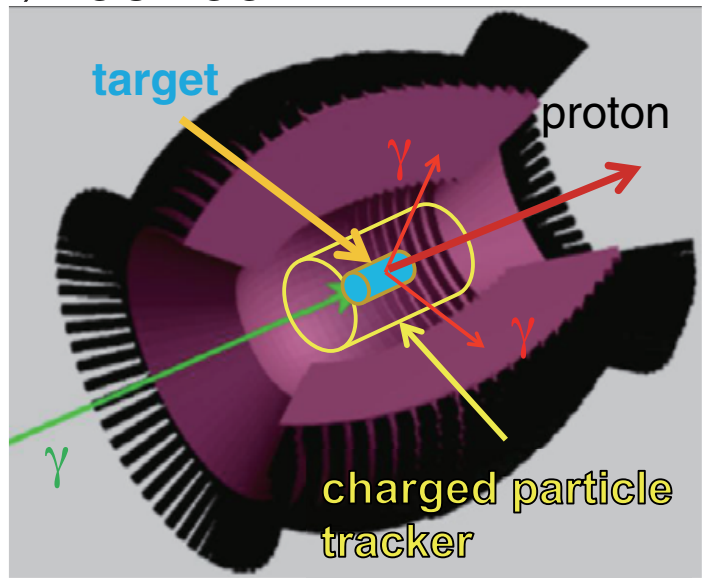

(b)

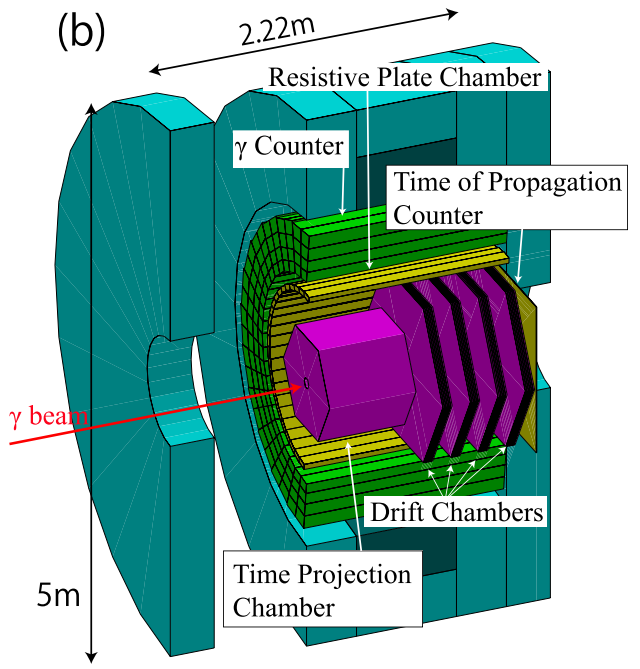

Figure 2. Schematic drawings of the BGO-EGG detector (a) and the LEPS2 solenoid magnetic spectrometer (b).

Two different experiments have been proposed at LEPS II, the one is the experiment using a photon detector, BGO-EGG, and the other is the large solenoid magnetic spectrometer. The BGO-EGG consists of $1320 \mathrm{Bi}_{4} \mathrm{Ge}_{3} \mathrm{O}_{12}$ (BGO) crystals which are arranged as "EGG"-shape. The polar-angle coverage is $24^{\circ}<\theta<144^{\circ}$ and thickness of each crystal is $\sim 20$ radiation lengths as shown in Fig. 2a. A cylindrical drift chamber and a planar drift chamber are installed inside and downstream of the BGOEGG, respectively, to measure directions of charged particles. In addition, a high resolution time-offlight counter which consists of resistive plate chambers is located $\sim 12 \mathrm{~m}$ downstream from the target, which can measure the energy of a forward-going proton with $\sim 20 \mathrm{MeV}$ resolution (r.m.s.) at the $2 \mathrm{GeV}$ energy. The commissioning run using a part of the detector system has started in late 2013, and the physics run will start from spring in 2014. Various meson photoproduction from a liquid hydrogen or nuclear targets will be measured using the linearly polarized beam.

Figure $2 b$ shows the LEPS II solenoid magnetic spectrometer. The solenoid magnet which was used for the kaon rare-decay experiment at Brookhaven National Laboratory (BNL-AGS, E787/E949) in U.S. was shipped to SPring-8 in Japan. The inner diameter and length are $2.96 \mathrm{~m}$ and $2.22 \mathrm{~m}$, respectively, 
and the magnetic field of $1 \mathrm{~T}$ is applied. The barrel electromagnetic calorimeter has also been shipped from BNL to be used together with the solenoid magnet. The detectors for charged particle tracking and particle identification (PID) are newly constructed. A time projection chamber and four drift chambers are employed for the tracking. In order to identify low momentum kaons, a high resolution time-of-flight counter consists of resistive plate chambers is used. For the PID of high momentum particles, aerogel Čerenkov counters and time of propagation counters $[14,15]$ will be developed. Detector development is underway, and physics run will start in near future.

\section{Summary}

A new GeV-photon beamline at SPring-8, LEPS II, has been constructed. We have successfully observed the first beam at LEPS II in early 2013. A clear BCS spectra and an excellent beam profile have been confirmed. The development of detectors for the solenoid magnetic spectrometer is underway. The commissioning run of a photon detector, BGO-EGG, has started in late 2013, and physics data will start in spring of 2014 .

\section{References}

[1] N. Muramatsu et al. Nucl. Inst. Methods. A 737184 (2014)

[2] F. Cooper Phys. Rev. 1701602 (1968)

[3] S.H. Hwang et al. Phys. Rev. Lett. 108, 092001 (2012)

[4] R.H. Dalitz, T.C. Wong and G. Rajasekaran Phys. Rev. 153, 1617 (1967)

[5] N. Kaiser, P.B. Siegel and W. Weise, Nucl. Phys. A 594, 325 (1995)

[6] J.C. Nacher, E. Oset, H. Toki and A. Ramos, Phys. Lett. B 455, 55 (1999)

[7] M. Niiyama et al., Phys. Rev. C 78, 035202 (2008)

[8] K. Moriya et al. [CLAS Collaboration], Phys. Rev. C 88, 045201 (2013)

[9] T. Hyodo, A. Hosaka, M.J. Vicente Vacas, E. Oset, Phys. Lett. B 593, 045201 (2013)

[10] T. Nakano et al., Phys. Rev. Lett. 91, 012002 (2003)

[11] T. Nakano et al., Phys. Rev. C. 79, 025210 (2009)

[12] B. McKinnon et al., Phys. Rev. Lett. 96, 212001, (2006)

[13] Y. Kato, Few-Body Syst. 541245 (2013) (FB20 Proceedings)

[14] M.S. Atiya et al., Nucl. Instrum. Meth. A 321, 129 (1992)

[15] M. Akatsu et al., Nucl. Instrum. Meth. A 440, 124 (2000) 